\title{
Correlation of Vertical Cup Disc Ratio and Matrix FDT Mean Deviation in Primary Open Angle Glaucoma Patients
}

\author{
Nathaniel Godswill Inye ${ }^{1, \text { *, Pepple Godswill }}{ }^{2}$ \\ ${ }^{1}$ University of Port Harcourt Teaching Hospital, Port Harcourt, Nigeria \\ ${ }^{2}$ Rivers State University Teaching Hospital, Port Harcourt, Nigeria
}

Email address:

drnathy2013@gmail.com (N. G. Inye)

${ }^{*}$ Corresponding author

\section{To cite this article:}

Nathaniel Godswill Inye, Pepple Godswill. Correlation of Vertical Cup Disc Ratio and Matrix FDT Mean Deviation in Primary Open Angle Glaucoma Patients. International Journal of Ophthalmology \& Visual Science. Vol. 6, No. 1, 2021, pp. 17-21.

doi: $10.11648 /$ j.ijovs.20210601.13

Received: November 18, 2020; Accepted: December 11, 2020; Published: February 10, 2021

\begin{abstract}
Objectives: To evaluate the correlation between glaucomatous structural change using vertical-cup-disc ratio of the optic nerve head and functional loss measured by Frequency Doubling Technology perimetry. Materials and methods: It was a retrospective observational clinic-based study of 77 newly diagnosed Primary Open Angle Glaucoma (POAG) patients from a Private Eye clinic in a suburb of Port Harcourt city, Nigeria. They underwent clinical fundal examination with slit-lamp biomicroscope (Perkin's slit-lamp) with 90D (Volk) lens; and Frequency Doubling Technology perimetry 242 threshold (Humphrey Matrix 800, 2011 Carl Zeiss Meditec, Dublin, California). The Vertical-cup-disc-ratio was assessed for each eye and the Mean deviation calculated for each eye. The relationship between VCDR and mean deviation was evaluated using Pearson's correlation coefficient regression analysis. Result: There were 24 males $(31.17 \%)$ and 53 females $(68.83 \%)$. The mean age of the patients was $56.65 \pm 14.33$ years with a range of 37-78 years. The mean VCDR was $0.83 \pm 0.09$ and $0.78 \pm 0.13$ for the right and left eyes, respectively while mean FDT mean deviation was $-13.99 \pm 9.33 \mathrm{Db}$ and 11.9.22 Db for the right and left eyes, respectively. The VCDR significantly correlated with FDT mean deviation (Pearson Correlation Coefficient, $\mathrm{r}=-0.923, \mathrm{r}^{2}=0.852, p$-value $=0.001$ for the right eye; $\mathrm{r}=-0.915, \mathrm{r}^{2}=0.836, p$-value $=0.001$ for the left eye. Conclusion: This study shows that at least $80 \%$ of VCDR correlated with the Mean deviation of matrix FDT amongst this cohort of POAG patients. it buttresses the fact that clinical evaluation of the optic nerve remains an indispensable tool in glaucoma diagnosis and can use to assess glaucoma severity in resource poor setting where visual field machine is not available.
\end{abstract}

Keywords: Correlation, Vertical-cup-disc-ratio, FDT Mean Deviation, Primary Open-angle Glaucoma

\section{Introduction}

Glaucoma is a chronic, progressive optic neuropathy, characterized by atrophy of the optic nerve and loss of retinal ganglion cells, resulting in progressive vision loss and Primary open angle glaucoma (POAG) is the most common variant [1]. In 2010, Quigley and Browman estimated that 44.7 million people worldwide suffered from primary open-angle glaucoma (POAG), and 4.5 million were blind, making POAG the most common type of glaucoma [2]. In 2015, Kapetanakis et al reported that 57.5 million people worldwide were affected by POAG [3]. Primary Open Angle Glaucoma development is associated with loss of tissue in the neuro-retinal rim of the optic disc and a consequent increase in the size of the optic cup [4].

Diagnosis of POAG requires an assessment of intraocular pressure, Open- normal appearing anterior chamber angle, characteristics signs of optic disc damage and visual function loss on perimetry. Clinical assessment of an optic disc is still considered the gold standard in the diagnosis of POAG, though the inter-observer agreement is notoriously variable. The vertical cup/disc ratio (CDR) has long been used in the assessment of the glaucoma patients, though the wide range of CDR values in the normal population limits its use. Cup size is related physiologically to disc size and pathologically to glaucomatous damage [5]. However, certain findings on 
examination are very suggestive of POAG and their presence even on a single observation can imply the presumptive diagnosis of glaucoma. Among these observations are nerve fibre layer (NFL) defects, optic nerve rim hemorrhages, and neuro-retinal rim notching or relative thinning.

The White-on-White Standard Automated Perimetry (SAP) is the gold standard for glaucoma diagnosis and monitoring of progression [6]. However, SAP only detects visual changes after substantive structural damage. Frequency doubling Technology (FDT) visual field test can substantially detect visual field changes in the early stages of the disease [7]. Humphrey Matrix FDT perimeter is a secondary generation FDT perimeter that utilizes additional test to improve the spatial resolution of visual field defects by using smaller targets with a higher spatial frequency and a lower temporal frequency [8,9]. Matrix FDT has a higher sensitivity in detecting early glaucomatous loss and better characterization of the pattern of visual field loss [10]. In addition, Matrix perimetry has been suggested to have additional benefits for monitoring subtle progression in glaucomatous field defects [11]. Several studies have shown that FDT has excellent results in detecting early, moderate, and advanced visual field loss in patients with glaucoma [7, 9-10, 12-13].

The relationship between the structural and functional deficits in glaucoma is a complex one [14-16]. Some studies have reported that functional deficit occurs before structural deficit $[17,18]$. However, other studies have shown that structural deficit occurs many years before manifestation of visual field defects [6,19-21].

Optic nerve head cupping is one the structural changes that occur in glaucoma [19]. Cup-disc ratio is a surrogate for the measurement of retinal nerve fiber loss as evidenced by thinning of the neuro-retinal rim [22]. Loss of retinal nerve fiber over time leads to visual field defects. The aim of this study is to evaluate the correlation between the structural changes using clinical subjective measures of the Vertical Cup-Disc Ratio (VCDR) and functional changes using the Mean deviation on Matrix FDT perimetry in patients with Primary Open Angle Glaucoma (POAG).

\section{Materials and Methods}

It was a retrospective observational clinic-based study correlating the Mean deviation on Matrix FDT perimetry to the Vertical Cup Disc Ratio (VCDR) of newly diagnosed Primary Open Angle Glaucoma (POAG) patients managed in an Eye Unit of a private hospital Life forte Specialist Hospital in the suburb of Port Harcourt, Nigeria.

Seventy-seven newly diagnosed POAG patients seen over a period of 6 years, January 2015 to August 2020 who met the study criteria were included in this study. The Ethical Committee of the University of Port Harcourt Teaching Hospital gave the approval for the study and the tenets of Helsinki Declaration involving the use of human subjects were adhered to.

Data collected included patients' demographic information (sex, gender, ethnicity, educational status); clinical information obtained included intraocular pressure (IOP) measured with handheld Perkin's Applanation tonometer (PAT), gonioscopy using Volk 3-mirror indirect gonio lens, Vertical Cup Disc Ratio (VCDR) assessed by stereoscopic examination using Keeler's slit-lamp biomicroscope with Volks 90D lens. All the eyes were dilated with Mydriacyl 1\% and Phenylephrine $2.5 \%$ before the fundus examination. All the patients were examined by only one examiner (GIN).

Patients with dense cataract and concomitant retina diseases such as diabetic retinopathy, retinal vascular occlusive diseases were excluded.

Glaucoma was defined by the presence of typical optic neuropathy with associated visual field defect demonstrated in at least two consecutive reliable examinations using Matrix FDT 24-2 threshold (Humphrey Matrix 800, 2011 Carl Zeiss Meditec, Dublin, CA). A trained Optometrist performed all the visual field testing. The Mean deviation was computed and used to classify the visual field loss into Mild (MD $\geq-6$ decibel), Moderate (MD $=-6$ to -12 decibel) and Advanced glaucoma (MD $<-12$ decibel). Open anterior chamber angle was defined as visualization of at trabecular meshwork in at least 3 quadrants using the Shaffer's grading on indirect gonioscopy.

\section{Statistical Analysis}

All data were cross checked for accuracy and entered in a proforma and were analyzed using commercially available statistical data management software- Statistical Package for Social Sciences (IBM-SPSS) version 25. Continuous variables were illustrated in the form of mean \pm standard deviation (SD) and categorical variables were shown in the form of frequency and percent. Pearson's correlation coefficient was used to investigate the relation between the mean deviation and VCDR. P value less than 0.05 was considered statistically significant.

\section{Result}

There were 24 males $(31.17 \%)$ and 53 females $(68.83 \%)$. The mean age of the patients was $56.65 \pm 14.33$ years with a range of $37-78$ years. Over $81 \%$ of the patients had formal education. Details of the demographics are as shown in table 1.

Table 1. Socio-demographic Characteristics.

\begin{tabular}{|c|c|c|}
\hline \multirow{2}{*}{ Characteristics } & Frequency & \multirow{2}{*}{ Percentage $(\%)$} \\
\hline & $(n=77)$ & \\
\hline \multicolumn{3}{|l|}{ Age } \\
\hline $30-39$ & 12 & 15.58 \\
\hline $40-49$ & 10 & 12.99 \\
\hline $50-59$ & 23 & 29.87 \\
\hline $60-69$ & 11 & 14.29 \\
\hline $70-79$ & 21 & 27.27 \\
\hline Mean [Range] & \multirow{2}{*}{\multicolumn{2}{|c|}{$56.65 \pm 14.33$ years $[37-78]$}} \\
\hline Sex & & \\
\hline Male & 24 & 31.17 \\
\hline
\end{tabular}




\begin{tabular}{lll}
\hline Characteristics & Frequency & Percentage (\%) \\
\cline { 2 - 3 }$(\mathbf{( n = 7 7 )}$ & 68.83 \\
\hline Female & 53 & 18.18 \\
Educational Status & & 27.27 \\
None & 14 & 54.55 \\
Secondary & 21 & \\
Tertiary & 42 & 25.97 \\
Ethnicity & & 20.78 \\
Ogoni & 20 & 19.48 \\
Ikwerre & 16 & 15.58 \\
Efik/Ekpeye/Ibibio & 15 & 7.79 \\
Ijaw & 12 & 5.19 \\
Ibo & 6 & 5.19 \\
Ogba & 4 & \\
Yoruba & 4 &
\end{tabular}

Mean Intraocular pressure, Mean Deviation and VCDR

The mean value of the Mean Deviation of Matrix FDT perimetry indicated that most of the patients have moderateto-Advanced disease in both eyes. Similarly, the mean VCDR showed similar pattern in both eyes of the patients as shown in table 2. The mean intraocular pressure for the right eye (RE) and left eye (LE) was as shown in table 2.

Table 2. Mean CVF Mean deviation, VCDR and IOP

\begin{tabular}{lll}
\hline Characteristics & Mean \pm SD & Range $(\mathbf{m i n}-\mathbf{m a x})$ \\
\hline & $(\mathbf{n}=\mathbf{7 7})$ & \\
\hline Mean Deviation $(\mathrm{dB})$ & & $-26.68,-1.37$ \\
RE & $-13.99 \pm 9.33$ & $-25.43,-1.52$ \\
LE & $-11.88 \pm 9.22$ & \\
VCDR & & $0.7-0.96$ \\
RE & $0.83 \pm 0.09$ & $0.60-0.96$ \\
LE & $0.78 \pm 0.13$ & \\
IOP $(\mathrm{mmHg})$ & & $13-41$ \\
RE & $23.48 \pm 10.91$ & $14-35$ \\
LE & $21.69 \pm 8.15$ & \\
\hline
\end{tabular}

Correlation between mean VCDR and FDT Mean Deviation

A statistically significantly strong linear but negative correlation exists between structural damage (VCDR) and visual field loss measured by the Mean deviation from FDT Matrix perimeter. For as the mean VCDR is increasing, mean deviation is decreasing: $\mathrm{RE}(\mathrm{r}=-0.923)$, LE $(\mathrm{r}=-0.915)$; $(p=0.001)$. R-Square $\left(\mathrm{r}^{2}\right)$ is between 0.836-0.852, showing that between $83-85 \%$ of the VCDR values are correlating with the visual field loss as measured by the mean deviation (Table 3, Figures 1 and 2)

Table 3. Correlation between VCDR and the mean deviation (MD) in decibel from Matrix FDT.

\begin{tabular}{lll}
\hline VCDR & CVF MD & \\
\hline \multirow{3}{*}{ RE } & RE & \\
& The Pearson correlation coefficient, $\mathrm{r}$ & -0.923 \\
& R-Square $\left(\mathrm{r}^{2}\right)$ & 0.852 \\
& $p$-value & $0.001^{*}$ \\
& LE & \\
LE & The Pearson correlation coefficient, $\mathrm{r}$ & -0.915 \\
& R-Square $\left(\mathrm{r}^{2}\right)$ & 0.836 \\
& p-value & $0.001^{*}$ \\
\hline
\end{tabular}

* Statistically significant $(\mathrm{p}<0.05)$

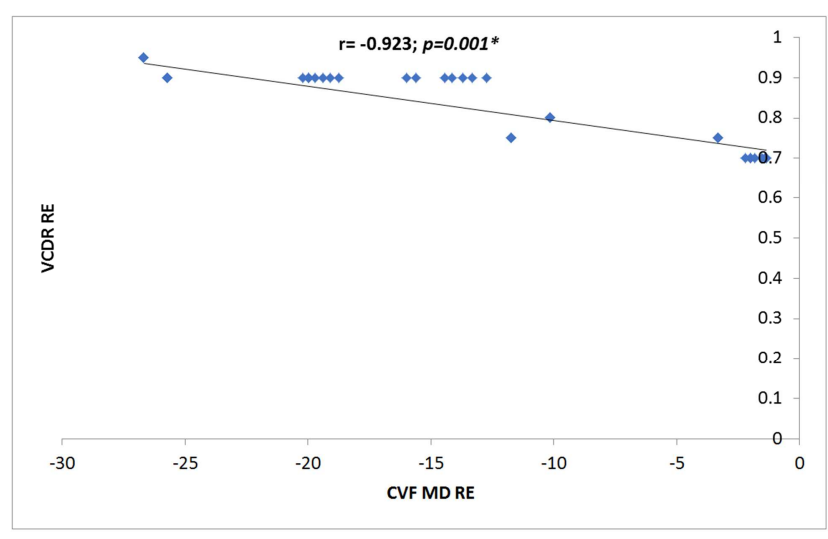

Figure 1. Correlation between VCDR and Matrix FDT Mean deviation RE.

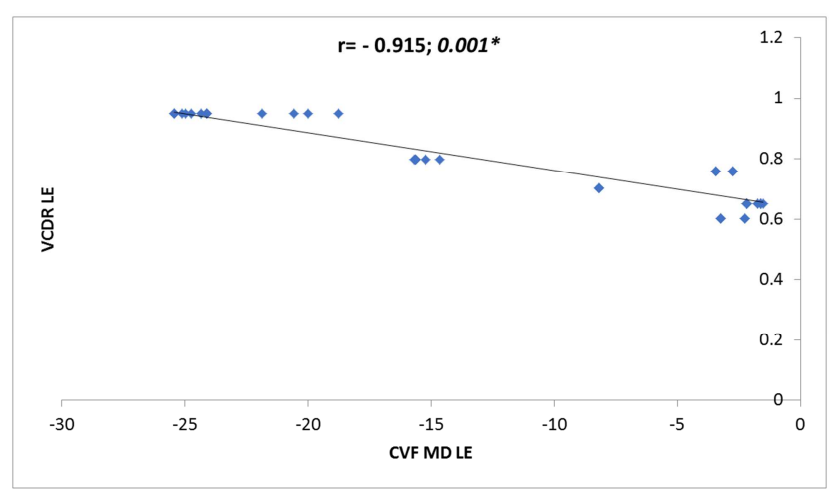

Figure 2. Correlation between VCDR and Matrix FDT Mean deviation LE.

\section{Discussion}

The optic cup has been recognized as an important morphological feature of the glaucomatous eye and the most used sign for the diagnosis of glaucomatous damage is an increased cup-disc ratio [23]. Cupping is an important early sign and progression of cupping has been observed in glaucoma before the onset of visual field damage [19, 24-25].

In low resource setting, the availability of imaging facilities such as Optical coherent tomography (OCT), stereoscopic fundus photograph etc., is scarce. So, diagnosis of glaucoma is essentially based on thorough clinical evaluation of the optic nerve head. it has been noted that corrected VCDR for size including neuro-retinal rim area, rim-to-disc area ratio, are the most valuable optic disc variables for early detection of glaucomatous optic disc damage [26].

In evaluating structure-function relationship, Frequency Doubling Technology has been found to have a higher correlation with structural damage than standard automated perimetry [27,28]. In this study, we correlated the VCDR and mean deviation from Matrix FDT perimetry and found a strong but negative correlation between the two variables in both eyes of the subjects ( $\mathrm{RE}(\mathrm{r}=-0.923), \mathrm{LE}(\mathrm{r}=-0.915)$; $(p=0.001)$. R-Square $\left(\mathrm{r}^{2}\right)$ is between $0.836-0.852$, showing that between $83-85 \%$ of the VCDR values are correlating with the visual field loss as measured by the mean deviation). In a study involving disc suspect and ocular hypertensive 
patients by Kaushit and colleagues, they found out that there was no correlation between VCDR and FDT Mean deviation in Glaucoma suspects [29]. However, they observed a significantly but negative correlation between VCDR and FDT Mean deviation among the Ocular Hypertensives. Using Visual Field Index (VFI) from Standard Automated Perimetry (SAP), Iutaka et al observed a significant linear correlation $(\mathrm{R}=-0.179, \mathrm{P}=0.012)$ with cup-disc ratio among POAG patients [30]. In these studies, the VCDR was assessed using optical coherence tomography (OCT). The study by Kaushit et al did not include glaucoma patients and therefore makes comparison with our study a bit difficult We could not find any study comparing these two variables in POAG patients as done in our study. This is one of the strengths of this study. Optic nerve head size varies with attendant variation in the VCDR in individuals. We did not correct the VCDR for size of the optic nerve head and this is a major limitation of this study.

In our study, most of the patients presented with moderate to severe glaucoma (FDT Mean deviation: RE -13.99 \pm 9.33 decibel, LE-11.88 \pm 9.22 decibel). The association between structural and functional measures has been found to increase with severity of the disease and this may account for the significant correlation observed [16].

Most studies on structure-function relationship focus on retinal nerve fiber layer (RNFL) thickness measured with OCT and perimetric variables [14-16, 21, 28-30]. The correlation between retinal nerve layer thickness thickness and perimetric mean deviation varies according to the type of OCT used [31].

Our study has helped to enhance the usefulness of verticalcup-disc ratio (VCDR) in the diagnosis of glaucoma especially in low resource setting where access to Imaging facilities may be limited.

\section{Conclusion}

This study shows that at least $80 \%$ of VCDR correlated with the Mean deviation of matrix FDT amongst this cohort of POAG patients. It helps to buttress the fact that properly clinical evaluation of the optic nerve head remains an indispensable tool in glaucoma diagnosis and can be use in assessing glaucoma severity in resource poor setting where visual field machine is unavailable,

\section{References}

[1] Leske MC. Open-angle glaucoma - an epidemiologic overview. Ophthalmic Epidemiol. 2007;14(4):166-172

[2] Quigley HA, Broman AT. The number of people with glaucoma worldwide in 2010 and 2020. Br J Ophthalmol. 2006;90(3):262-267.

[3] Kapetanakis VV, Chan MP, Foster PJ, Cook DG, Owen CG, Rudnicka AR. Global variations, and time trends in the prevalence of primary open angle glaucoma (POAG): a systematic review and meta-analysis. $\mathrm{Br} \mathrm{J}$ Ophthalmol. 2016;100(1):86-93.

[4] Betz P, Camps F, Collignon-Brach J, et al. Biometric study of the disc cup in open-angle glaucoma. Graefes Arch Clin Exp Ophthalmol1982; 218:70-74.

[5] Garway-Heath DF, Ruben S, Viswanathan A, Hitchings RA. Vertical cup/disc ratio in relation to optic disc size: Its value in the assessment of the glaucoma suspect. Br J Ophthalmol 98; 82 (10): 1118-1124.

[6] Sommer A, Katz J, Quigley HA et al. "Clinically detectable nerve fiber atrophy precedes the onset of glaucomatous field loss," Arch Ophthalmol 1991; 109(1):77-83.

[7] Johnson CA, Samuels JS, "Screening for glaucomatous visual field loss with frequency-doubling perimetry," Investigative Ophthalmology \& Visual Science. 1997; 38(2):413-425.

[8] Zarkovic A, Mora J, McKelvie J, Gamble G (2007) Relationship between second-generation frequency doubling technology and standard automated perimetry in patients with glaucoma. Clin Experiment Ophthalmol 35: 808-811.

[9] Spry PG, Hussin HM, Sparrow JM (2005) Clinical evaluation of frequency doubling technology perimetry using the Humphrey Matrix 24-2 threshold strategy. Br J Ophthalmol 89: 1031-1035.

[10] Johnson CA, Cioffi GA, Van Buskirk EM (1999) Frequency doubling technology perimetry using a 24-2 stimulus presentation pattern. Optom Vis Sci 76: 571- 581.

[11] Bozkurt B, Yilmaz PT, Irkec M (2008) Relationship between Humphrey 30-2 SITA Standard Test, Matrix 30-2 threshold test, and Heidelberg retina tomograph in ocular hypertensive and glaucoma patients. J Glaucoma 17: 203-210.

[12] Kim TW, Zangwill LM, Bowd C, Sample PA, Shah N, et al. Retinal nerve fiber layer damage as assessed by optical coherence tomography in eyes with a visual field defect detected by frequency doubling technology perimetry but not by standard automated perimetry. Ophthalmology 2007; 114: 1053-1057.

[13] Artes PH, Hutchison DM, Nicolela MT, LeBlanc RP, Chauhan BC (2005) Threshold and variability properties of matrix frequency-doubling technology and standard automated perimetry in glaucoma. Invest Ophthalmol Vis Sci 46: 24512457.

[14] El Beltagi TA, Bowd C, Boden C, et al. Retinal nerve fiber layer thickness measured with optical coherence tomography is related to visual function in glaucomatous eyes. Ophthalmology. 2003; 110:2185-2191.

[15] Nilforushan N, Nassiri N, Moghimi S, et al. StructureFunction Relationships between Spectral-Domain OCT and Standard Achromatic Perimetry. Invest Opthalmol Vis Sci. 2012;:2740-2748.

[16] Leite MT, Zangwill LM, Weinreb RN, et al. StructureFunction relationships using the Cirrus Spectral Domain Optical Coherence Tomography and Standard Automated Perimetry. J Glaucoma. 2012; 21:49-54.

[17] Leske MC, Heijl A, Hyman L, et al. Early Manifest Glaucoma Trial: design and baseline data. Ophthalmology. 1999; 106:2144-153. 
[18] Miglior S, Zeyen T, Pfeiffer N, Cunha-Vaz J, Torri V, Adamsons I, European Glaucoma Prevention Study (EGPS) Group. Results of the European Glaucoma Prevention Study. Ophthalmology. 2005; 112:366-375.

[19] Pederson JE, Anderson DR. The Mode of Progressive Disc Cupping in Ocular Hypertension and Glaucoma. Arch Ophthalmol. 1980; 98:490-495.

[20] Hood DC, Kardon RH. A framework for comparing structural and functional measures of glaucomatous damage. Prog Retin Eye Res. 2007; 26:688-710.

[21] Kuang TM, Zhang C, Zangwill LM, et al. Estimating Lead Time Gained by Optical Coherence Tomography in Detecting Glaucoma before Development of Visual Field Defects. Ophthalmology. 2015; 122:2002-2009.

[22] Thomas R, Loibi K, Prarikh R. Evaluation of a glaucoma patient. Indian J Ophthalmol 2011; 59: 43-52.

[23] Ritch R, Shields M, Krupin TCaprioli J(1996) Quantitative measurements of the optic nerve head. in The glaucomas. 2 ed. eds Ritch R, Shields M, Krupin T (Mosby-Year Book, St Louis), pp 659-675.

[24] Sommer A, Pollack I, Maumenee AE. Optic disc parameters and onset of glaucomatous field loss. I. Methods and progressive changes in disc morphology. Arch Ophthalmol 1979; 97:1444-1448.

[25] Read RM, Spaeth GL. The practical clinical appraisal of the optic disc in glaucoma: The natural history of cup progression and some specific disc-field correlations. Trans Am Acad Ophthalmol Otolaryngol 1974; 78:255-267.

[26] Jonas JB, Bergua A, Schmitz-Valckenberg P, Papastathopoulos KI, Budde WM. Ranking of Optic Disc Variables for Detection of Glaucomatous Optic Nerve Damage. Invest Ophthalmol Vis Sci 2000; 41:1764-1773.

[27] Lamparter J, Russell RA, Schulze A, et al. Structure-Function Relationship between FDF, FDT, SAP, and Scanning Laser Ophthalmoscopy in Glaucoma Patients. Invest Opthalmol Vis Sci. 2012; 53:7553-9.

[28] Nathaniel GI, Ogunro A, Awoyesuku EA. The correlation between Frequency Doubling Technology Matrix Mean Deviation and Peripapillary Retinal Nerve Fibre thickness of newly diagnosed glaucoma patients. International Journal of Medical Ophthalmology 2020: vol 2 (1): 32-36.

[29] Kaushit S, Pandav SS, Ichhpujani P, Gupta A. Correlation of Frequency Doubling Perimetry with Retinal Nerve Fibre Layer thickness and Optic Disc Size in Ocular hypertensives and Glaucoma suspects. J Glaucoma 2011; 20: 366-370.

[30] Iutaka NA, Grochowski RA, Kasashara N. Correlation between Visual Field Index and other Functional and Structural Measures in Glaucoma Patients and Suspects. J Ophthalmics Vis Res 2017; 12: 53-57.

[31] Silva AL, Melo LA, Campos M, Tavares I. Correlation between peripapillary retinal nerve fibre layer thickness and visual field in glaucoma. Invest Ophthalmol Vis Sci 2013; 54: 2283. 